\title{
Arbor
}

\section{Influencia de la actividad física en la salud humana}

\author{
Juan Manuel Alonso Martín
}

Arbor CLXV, 650 (Febrero 2000), 187-201 pp.

El ejercicio físico es un agente importante en el mantenimiento de la salud y en la prevención de diversas enfermedades. El cuidado en la enfermedad coronaria, la hipertensión, la diabetes, la obesidad, el cáncer, la osteoporosis y el incremento de la salud mental se han relacionado con la actividad física y el ejercicio. En éste artículo se resumen los efectos de la realización de deporte en las enfermedades más comunes de la sociedad actual. Sin embargo, la mayoría de la población no practica actividad fisica. Por lo tanto, es preciso que los médicos prescriban más frecuentemente el ejercicio físico.

El ejercicio es un agente de importancia en el mantenimiento de la salud y en la prevención de diversas enfermedades ${ }^{10}$. El papel de la actividad física en la prevención y control de las enfermedades crónicas ha sido bien documentado. El cuidado en la enfermedad coronaria, la hipertensión, la diabetes, la obesidad, el cáncer, la osteoporosis y el incremento de la salud mental se han relacionado muy claramente con actividad física y el ejercicio ${ }^{5,12}$ (Tabla 1). Desdichadamente la mayoría de la población no practica actividad física. No obstante, el ejercicio se está empezando a utilizar más ampliamente en la prevención de las enfermedades que son más prevalentes. Aún así los médicos necesitan más formación en este campo con el objeto de que prescriban más frecuentemente la actividad física. 
TABLA 1. Enfermedades en las que se puede emplear el ejercicio físico como tratamiento o factor preventivo

$\begin{array}{ll}\text { Enfermedades Cardiovasculares } & \text { Cáncer de mama } \\ \text { Enfermedad Pulmonar Obstructiva Crónica } & \text { Cáncer de cólon } \\ \text { Enfermedad Renal } & \text { Artritis } \\ \text { Diabetes } & \text { Artrosis } \\ \text { Menopausia } & \text { Osteoporosis }\end{array}$

\section{Beneficios en el aparato cardiovascular}

El ejercicio físico junto con la dieta adecuada puede revertir una enfermedad cardíaca establecida. Más aún, la práctica deportiva mejora la función cardíaca, reduce o modifica varios de los factores de riesgo coronarios como la hipertensión, colesterol elevado, niveles bajos de lipoproteínas de alta densidad ligadas a colesterol (HDL) y la obesidad (Tabla 2). Así mismo, el ejercicio mejora el bienestar psico-social y en general aumenta la supervivencia ${ }^{5}$.

TABLA 2. Enfermedades cardiovasculares en las que se ha demostrado el beneficio de la práctica de actividad física

Infarto de miocardio

Arteriosclerosis

Enfermedad cerebro-vascular

Hipertensión

Alteraciones de los perfíles lipídicos

Los investigadores finlandeses Lakka et al mostraron en 1994, tras un estudio de cinco años, que se puede reducir el riesgo de infarto de miocardio con dos horas a la semana de ejercicio físico de mantenimiento ${ }^{9}$. En dicho estudio encontraron que aquellas personas que realizaron-ejercicio físico de acondicionamiento tal como andar, correr o nadar en el que aumentaron seis o más veces su metabolismo basal tuvieron un riesgo $60 \%$ más bajo de padecer infartos de miocardio ${ }^{9}$. Esta observación ha sido apoyada por varios estudios prospectivos llevados a cabo en Europa y Norteamérica realizados en grupos de individuos aparentemente sanos que fueron seguidos hasta durante 20 años. La mayoría de los estudios mostraron una relación 
inversa entre los niveles de actividad física y la incidencia de enfermedad coronaria ${ }^{4}$.

El grupo investigador integrado por Ornish et al demostraron en 1983 que la realización de ejercicio físico junto con una dieta pobre en grasa, abandono del tabaco y modificación del estrés disminuyeron el diámetro de la estenosis de las arterias coronarias. Con ello demostraron que la arteriosclerosis es reversible y entre otros factores, la práctica deportiva moderada puede contribuir a ello ${ }^{10}$. Otros trabajos de investigación en los que se incluyó el ejercicio dentro del régimen terapéutico documentaron en angiografías una regresión de las placas de ateroma en las arterias ${ }^{4}$.

La práctica de ejercicio físico vigoroso durante los primeros años que siguen a la consecución de la edad adulta confiere considerable protección frente a los accidentes cerebrovasculares, tanto tromboembólicos como hemorrágicos ${ }^{4,5}$. Este efecto es independiente de otros factores. Más aún, el ejercicio es esencial en restablecer la función tras un accidente cerebrovascular, un beneficio no compartido con el tratamiento farmacológico o la cirugía ${ }^{5}$.

El ejercicio aeróbico ayuda a controlar la presión sanguínea. Varios estudios realizados en humanos han demostrado que personas normo e hipertensivas exhiben una disminución post-ejercicio en los niveles basales de presión sanguínea ${ }^{4}$. Kasch en 1990 señaló que en los individuos que realizan de manera regular actividad física se producen elevaciones menos acusadas de las cifras tensionales con el envejecimiento ${ }^{10}$. La realización de actividad física es un tratamiento efectivo para la hipertensión leve y moderada y es una ayuda importante para el tratamiento de la hipertensión grave. Hagberg en 1990 realizó una revisión de literatura sobre este respecto y encontró que en el $65 \%$ de los trabajos se señalaba una disminución de alrededor de 8 a 10 $\mathrm{mm}$ de $\mathrm{Hg}$ en ambas presiones, la diastólica y la sistólica, con la realización de ejercicio regular ${ }^{4,10}$. Muchos pacientes que se impliquen en un programa de actividad física regular pueden reducir su tensión arterial sin necesidad de tomar medicamentos. El grado de reducción de la presión sanguínea depende del tipo, duración e intensidad del ejercicio así como los factores genéticos ${ }^{5}$. Los mecanismos hemodinámicos que explican el efecto hipotensor del ejercicio aeróbico pueden ser debidos a la disminución del gasto cardíaco y al descenso en la resistencia vascular periférica, así como la reducción de los niveles sanguíneos de adrenalina ${ }^{4}$. La actual recomendación para el control de la presión sanguínea elevada es la práctica deportiva aeróbica de mediana intensidad, definida como el ejercicio realizado entre 55 y 
$70 \%$ de la frecuencia cardiaca máxima, durante 30 a 40 minutos, 5 a 6 días a la semana ${ }^{1}$.

Las anomalías de los lípidos plasmáticos, tales como la elevación del nivel total de colesterol y triglicéridos o unos niveles reducidos de colesterol-HDL son factores de riesgo mayores de enfermedad cardiovascular ${ }^{5}$. Según el tipo, duración e intensidad del programa de ejercicios se producen algunas variaciones en los lípidos sanguíneos ${ }^{10}$. En general, el ejercicio regular reduce los niveles del colesterol total, de los triglicéridos y del colesterol-LDL y aumenta las cifras de colesterol-HDL ${ }^{5,10}$.

Sólo las personas con patología cardiaca previa están en riesgo de un accidente cardiovascular durante la práctica del ejercicio. No obstante, los beneficios derivádos de la práctica regular de ejercicio sobrepasan ampliamente los riesgos ${ }^{7}$. Aquellos pacientes que padecen enfermedad cardiovascular, antes de enrolarse en un programa de actividad física, deben someterse a una exhaustiva evaluación médica, y deben seguir escrupulosamente la vigilancia recomendada por los profesionales sanitarios.

\section{Ejercicio y Enfermedad Pulmonar Obstructiva Crónica (EPOC)}

Las personas que padecen enfermedad pulmonar restrictiva u obstructiva sufren una alteración en la función mecánica pulmonar así como una deficiencia en el intercambio pulmonar de gases ${ }^{4}$. La adición de ejercicio físico a los programas de rehabilitación de los pacientes que sufren EPOC consigue beneficios fisiológicos y psicológicos incluso en los que sufren severas obstrucciónes del flujo aéreo ${ }^{5}$. El ejercicio no puede revertir los déficits fisiológicos y estructurales de la EPOC, pero puede reducir la discapacidad de estos pacientes mejorando la resistencia, el flujo de la inspiración, el trabajo inspiratorio y, en consecuencia, la eficacia de la respiración y la tolerancia a la disnea, especialmente en pacientes con afectaciones graves ${ }^{4,11}$. La práctica deportiva mejora la eficacia de la respiración y disminuye la ansiedad, lo que mejora la ventilación. El incremento del lactato se reduce y se retarda con la realización habitual de práctica deportiva en los pacientes con EPOC. Los programas de ejercicio en los pacientes con EPOC retrasan la aparición de la disnea, ayudan a los pacientes a tolerarla y contribuyen a que éstos enfermos la teman menos ${ }^{11}$. 


\section{Influencia de la actividad física en la salud humana}

El primer paso de cualquier programa de ejercicio para los pacientes con EPOC es una cuidadosa evaluación para establecer el riesgo cardiaco y la capacidad de realizar actividad física. Con posterioridad es importante la elección de la modalidad de ejercicio. Dado que muchos de estos pacientes tienen un equilibrio inestable y se sienten más seguros sentados, la bicicleta estática es un modo extremadamente útil de realización de ejercicio. Los objetivos de intensidad de ejercicio deben ser muy modestos: si el paciente sólo es capaz de realizar ejercicio durante 5 minutos será suficiente. Lo más importante es la progresión muy lenta, teniendo siempre en cuenta que en este tipo de personas un pequeño incremento de volumen o intensidad del ejercicio puede dar como resultado grandes mejoras en la calidad de sus vidas ${ }^{11}$.

\section{Actividad física y enfermedad renal}

La calidad de vida y la supervivencia a largo plazo de los pacientes con enfermedad renal está reducida, debido, entre otras causas, a una arterosclerosis desarrollada de manera acelerada o a muerte por enfermedad cardiovascular. La capacidad funcional de esta población es pobre. Sólo la mitad de los pacientes que sufren insuficiencia renal crónica pueden caminar y la mayoría pasa la mayor parte del tiempo en reposo. Tienen reducida la capacidad de realizar ejercicio físico debido al aumento crónico de la urea en sangre, a las enfermedades cardiovascular y muscular, a la anemia y la hipervolemia, junto con la escasa motivación, la depresión y la fatiga. La mayoría de los pacientes sufren hipertensión, reducción de las lipoproteínas de alta densidad, hiperinsulinemia y arteriosclerosis. Por ello, aquellos pacientes que sufren enfermedad renal y que se encuentren en mejor estado físico pueden ser enrolados en un programa de ejercicio físico para mejorar la función cardiovascular, reducir la presión sanguínea, mejorar el metabolismo de los lípidos y la glucosa, incrementar la fuerza muscular, mejorar la función hematológica y el estado psicológico. Antes de iniciar la actividad física el paciente debe ser sometido a un examen médico completo y a una prueba de esfuerzo. Una vez que se establece la medicación, el régimen de dialisis y el régimen dietético, los pacientes pueden iniciar la practica de deportes aeróbicos a intensidades leves (caminar, nadar, o montar en bicicleta) con estrecha vigilancia médica ${ }^{4}$.

\section{Beneficios en la diabetes}

Las personas que padecen diabetes tienen ciertas desventajas para practicar ejercicio físico: poseen depósitos de glucógeno inferiores, una 
glicólisis reducida y menor producción de lactato, sus músculos capturan menos glucosa y, en cambio, liberan la glucosa a mayor velocidad por aumento de la glucogenolísis ${ }^{20}$.

No obstante, el ejercicio es un factor principal en el control de la diabetes junto con la dieta y la insulina y es particularmente importante para las personas que padecen diabetes tipo II o no-insulino dependiente. La literatura médica indica que el ejercicio tiene capacidad para mejorar la tolerancia a la glucosa y reducir la necesidad de insulina en pacientes con tolerancia alterada a la glucosa ${ }^{4}$.

Las bases fisiológicas son las siguientes: un músculo esquelético en contracción puede multiplicar por treinta y cinco la captación de glucosa $^{2,4,10}$. A continuación del ejercicio, se incrementa la tolerancia a la glucosa durante un tiempo variable, dependiendo de la insulina y de la actividad contráctil. Parece que los músculos ayudan a regular el transporte de la glucosa estimulado por la contracción ${ }^{2}$.

Por tanto, en cualquier estado en el que exista una alteración de la sensibilidad a la insulina, la actividad física es una lógica intervención terapéutica ${ }^{2}$. De hecho, se puede conseguir el control sanguíneo de la glucosa en pacientes con diabetes tipo II sólo con la práctica regular de ejercicio, la perdida de peso y una dieta con reducción de la ingesta calórica ${ }^{20}$. En efecto, el control glicémico es mejorado con el ejercicio en pacientes con diabetes mellitus tipo II menores de 55 años, en pacientes tratados con dieta y en aquellos con un control metabólico bueno ${ }^{6}$. Incluso las mujeres que padecen diabetes gestacional que eran activas físicamente previamente a quedar embarazadas, se benefician del mantenimiento de una actividad física moderada durante la gestación. Es más, aquellas mujeres sedentarias previamente sanas que no tengan contraindicaciones médicas $u$ obstétricas para la práctica del ejercicio durante el embarazo, pueden iniciar una actividad física de baja intensidad ${ }^{2}$.

La práctica regular de ejercicio en los pacientes con diabetes tipo II, puede ayudar a estabilizar diabetes pobremente controladas, reduce el peso, disminuye los niveles sanguíneos de glucosa, mejora los perfiles lipídicos sanguíneos y reduce la tensión sanguínea ${ }^{5,10}$. Adicionalmente el ejercicio incrementa la efectividad de la insulina y del metabolismo de los azúcares ${ }^{5}$. Todos estos beneficios rebajan la incidencia de enfermedades cardiovasculares y decrece la aparición de complicaciones como la angiopatía del cerebro, del corazón, del riñón y de los miembros inferiores, la neuropatía y la retinopatía ${ }^{5,20,21}$.

Así mismo, y por el mismo mecanismo descrito anteriormente, los pacientes que padecen diabetes tipo I, o insulino-dependiente, pueden 


\section{Influencia de la actividad física en la salud humana}

reducir la dosis necesaria de insulina practicando ejercicio físico. Sin embargo, en este tipo de pacientes el ejercicio puede llevar a complicaciones, por lo que deben tener especial cuidado con su ingesta de carbohidratos y han de vigilar sus niveles de glucosa sanguínea y la dosis de insulina antes y después de realizar ejercicio ${ }^{4,20}$. Los pacientes de diabetes tipo I deben tener siempre disponibles tiras medidoras de glucosa y algún alimento rico en una forma de carbohidratos rápidamente absorbible ${ }^{4}$.

El programa de ejercicio ideal para pacientes diabéticos de cualquiera de los dos tipos de diabetes está integrado principalmente por actividad de tipo aeróbico realizada de tres a cinco veces a la semana durante 30 minutos a una intensidad aproximada de $50 \%-70 \%$ del $\mathrm{VO}_{2 \max }{ }^{20}$. El programa podría incluir además ejercicios de fuerza ${ }^{6}$. En el caso de las mujeres que padecen diabetes gestacional, la prescripción debe ser individualizada y llevada a cabo bajo cuidadosa supervisión médica ${ }^{2}$.

Numerosos estudios descriptivos han demostrado que la prevalencia de Diabetes Mellitus tipo II es más elevada en individuos urbanos inactivos que en poblaciones rurales activas. Más aún, otros estudios han mostrado que la prevalencia de la Diabetes Mellitus tipo II es mayor entre los individuos sedentarios que en los activos, independientemente de la edad o del índice de masa corporal. Dos estudios epidemiológicos de poblaciones numerosas han señalado que el riesgo de desarrollar diabetes tipo II es menor en aquellos sujetos que se ejercitan regularmente ${ }^{4}$.

\section{Ejercicio y obesidad}

La cantidad de peso graso es un indicador útil de salud, así como también una señal temprana de alarma de múltiples enfermedades graves. En la obesidad, trastorno metabólico en el que intervienen múltiples factores como las modificaciones del metabolismo, las alteraciones hormonales y la herencia entre otras, se produce un aumento del peso $\mathrm{y}$ especialmente un incremento del porcentaje del peso graso ${ }^{4,10}$. El exceso de peso, se dé o no en la obesidad, es un factor de riesgo para la enfermedad cardiovascular, la diabetes, múltiples cánceres (mama, próstata, colon, útero, etc.) y muerte prematura por otras causas ${ }^{5}$. Es probable que la inactividad física contribuya al desarrollo de la obesidad en ciertos individuos mientras que la actividad física regular 
la previene en otros. No obstante, como se citó anteriormente, la inactividad física no es la única causa de la obesidad ${ }^{4}$.

La práctica de actividad física incrementa el gasto calórico total diario. En parte esto es debido al aumento del gasto energético durante la propia actividad física. Con el mantenimiento de la práctica regular de ejercicio puede incrementar la masa libre de grasa lo que, a su vez, aumenta el gasto calórico basal. El ejercicio aumenta la oxidación lipídica y produce generalmente cierta reducción de los depósitos grasos. La perdida de peso graso es mayor cuanto mayor sea la actividad física realizada y cuanto mayor sea el peso graso de la persona que inicie el ejercicio físico ${ }^{4}$.

El ejercicio físico habitual y constante es una estrategia esencial para conseguir y mantener un peso ideal. La dieta, es básica, pero no se puede confiar en ella solamente para conseguir perdida y mantenimiento del peso. La medida más eficaz para el tratamiento del exceso de peso y la obesidad es la asociación del ejercicio físico y la dieta hipocalórica equilibrada ${ }^{5,10}$. La pérdida de peso producida sólo por el ejercicio no da los mismos resultados que si se asocia a restricción calórica ${ }^{4}$.

\section{Menopausia}

El ejercicio físico puede afectar profundamente el periodo de la menopausia mejorando la calidad de vida. El ejercicio disminuye los síntomas de la menopausia y los factores de riesgo de la enfermedad coronaria, reduce el riesgo de osteoporosis, fortalece la musculatura pélvica y mejora el bienestar ${ }^{8,15}$.

Se ha demostrado que los síntomas vasomotores (enrojecimiento facial) son menos frecuentes en las mujeres post-menopáusicas físicamente activas. Sin embargo, hasta la fecha no se ha demostrado que la práctica de ejercicio alivie dichos síntomas ${ }^{15}$.

Por otro lado, las mujeres en edad menopáusica se pueden beneficiar de varios efectos positivos que causa la práctica regular del ejercicio físico. Entre otros, el aumento del Colesterol HDL y el descenso del colesterol LDL, disminución de la perdida de la masa ósea causada por la edad y en el plano psicológico, reducción de la ansiedad, de la tensión, de la depresión y de la fatiga ${ }^{8}$.

Las recomendaciones de ejercicio para las mujeres menopáusicas deben ir encaminadas a la realización de àctividad aeróbica que incluya ejercicios que supongan soporte del peso corporal (p.ej.: marcha, carrera, 
danza aeróbica) y la práctica de entrenamiento de fuerza al menos tres veces a la semana durante 20 a 30 minutos ${ }^{8,15}$. Para mantener la flexibilidad, deben realizarse ejercicios apropiados después de cada sesión de entrenamiento aeróbico o de fuerza.

\section{Cáncer de mama en la mujer}

Entre los factores de riesgo del cáncer de mama se pueden citar la herencia, la acumulación de ciclos ovulatorios y la inactividad física. Las mujeres que realizan ejercicio físico 4 horas a la semana reducen el riesgo de padecer cáncer de mama en más de un $50 \%$ comparadas con mujeres inactivas. La práctica regular de sólo 1 a 3 horas de ejercicio reduce el riesgo alrededor de un $30 \%{ }^{3,14}$. Los beneficios son más evidentes en aquellas mujeres que han practicado actividad física tras la pubertad y entre los 20 y 25 .años. El ejercicio puede proteger las mamas alterando los niveles hormonales y disminuyendo la ovulación. Dado que un número bajo de ciclos ovulatorios puede proteger frente al cáncer de mama, el ejercicio moderado iniciado en la adolescencia podría ser una forma importante de prevenir el cáncer de mama ${ }^{14}$.

\section{Otros cánceres}

Existen evidencias científicas consistentes de que la actividad física reduce el riesgo de cáncer de colon ${ }^{4,5}$. Los posibles mecanismos citados son la aceleración del tránsito intestinal que disminuiría el tiempo de contacto de los agentes carcinogénicos contenidos en las heces; el aumento de la síntesis de prostaglandinas que disminuiría el índice de división de las células del colon, a la vez que estimularía el peristaltismo; y la estimulación de la secreción de las hormonas intestinales que también aumentaría el peristaltismo intestinal y disminuiría los ácidos biliares ${ }^{4,10}$.

Se piensa que el ejercicio prolongado de tipo aeróbico, al provocar cierta disminución en los niveles sanguíneos de testosterona puede tener un efecto protector frente al cáncer de próstata en el hombre. No obstante, son necesarios estudios que confirmen estas teorías ${ }^{4,10}$.

\section{Beneficios en la artritis}

Las enfermedades osteoarticulares inflamatorias o degenerativas producen gran incapacidad en la actividad diaria. Los pacientes que 
sufren artritis reumatoide o artrosis degenerativa se pueden beneficiar de varios efectos positivos del ejercicio físico. Éste mejora la resistencia, fortalece los músculos e incrementa la flexibilidad articular y el rango de movimiento, reduce el dolor y facilita el desarrollo de las actividades domiciliares, laborales y sociales ${ }^{5,10}$.

\section{Beneficios del ejercicio en la osteoporosis}

La osteoporosis es una enfermedad ósea caracterizada por la pérdida de sales minerales y cuya incidencia suele ser mucho más elevada en las últimas etapas de la vida, especialmente en las mujeres. Se ha podido comprobar que el ejercicio regular puede prevenir y controlar la osteoporosis, previniendo o revirtiendo la perdida de masa ósea ${ }^{5}$. Se ha podido comprobar que el ejercicio puede ser mayoritariamente efectivo en aumentar la masa ósea en adultos jóvenes y en el mantenimiento de la mása ósea en los adultos maduros ${ }^{4}$. De hecho, las personas involucradas en actividades físico-deportivas poseen mayor densidad ósea que las sedentarias, al mismo tiempo que en aquellas la desmineralización del hueso se lleva a cabo más lentamente ${ }^{10}$. Los datos obtenidos de diversos estudios indican que las personas activas de menos de cincuenta años de edad, poseen un $8 \%$ más de mása ósea en las mujeres y un $10 \%$ más en los hombres ${ }^{4}$. El efecto positivo del ejercicio en el hueso se observa también en mujeres postmenopáusicas y en hombres mayores. En mujeres postmenopáusicas físicamente activas se observa hasta un $7 \%$ más de mása osea que en las sedentarias de la misma edad. En general, aquellos individuos con menor mása ósea tienen mayor margen de mejora con la práctica de actividad física. La participación de mujeres mayores en programas de actividad física durante periodos prolongados supone un ligero aumento de la mása ósea a nivel espinal. Puede haber un cierto efecto protector del ejercicio físico sobre las facturas osteoporóticas, por disminución del riesgo a padecerlas por incremento en la densidad ósea, cambios en la microarquitectura ósea, o disminución de la posibilidad de padecer caídas ${ }^{4}$.

El ejercicio posee un efecto osteogéncio local ${ }^{4}$. Así, los ejercicios que soportan el peso corporal, como andar y correr, o los que suponen tracción o carga sobre los huesos como los ejercicios de pesas son los más beneficiosos para la protección frente a la pérdida de mineral óseo ${ }^{10}$. Por otro lado, debe haber un incremento progresivo de la intensidad del ejercicio para continuar la mejoría. Hay que tener en 


\section{Influencia de la actividad física en la salud humana}

cuenta, además, que los efectos positivos se pierden en cuanto se deja de practicar la actividad física ${ }^{4}$.

\section{Actividad física, función inmunitaria e infección}

Los personas muy activas pueden estar en riesgo de padecer varias infecciones debido a una situación de inmunosupresión causada por el entrenamiento intenso y la participación en competiciones ${ }^{4}$.

Recientemente se está prestando mucha atención al estrés físico y psicológico como factores que intervienen en la función y estado inmunitario y en las infecciones de vias respiratorias altas. La relación entre este tipo de infecciones y el ejercicio se pueden describir como una curva jota (figura 1). El riesgo de infecciones respiratorias es menor en las personas que practican ejercicio moderado por una estimulación de los mecanismos de defensa específicos e inespecíficos ${ }^{4,16}$. Por otro lado, el riesgo es más alto en los practicantes de deporte que realizan entrenamientos intensos y exhaustivos ya que este tipo de actividad parece que debilita los mecanismos de defensa ${ }^{4,16}$.

Figura 1. Curva Jota. Relación entre el nivel de ejercicio físico y la función inmunitaria. Modificado por Shepard, R., Pang, N. y DiNubile, N. Phys Sportsmed, 1999; 27 (6): 47-71

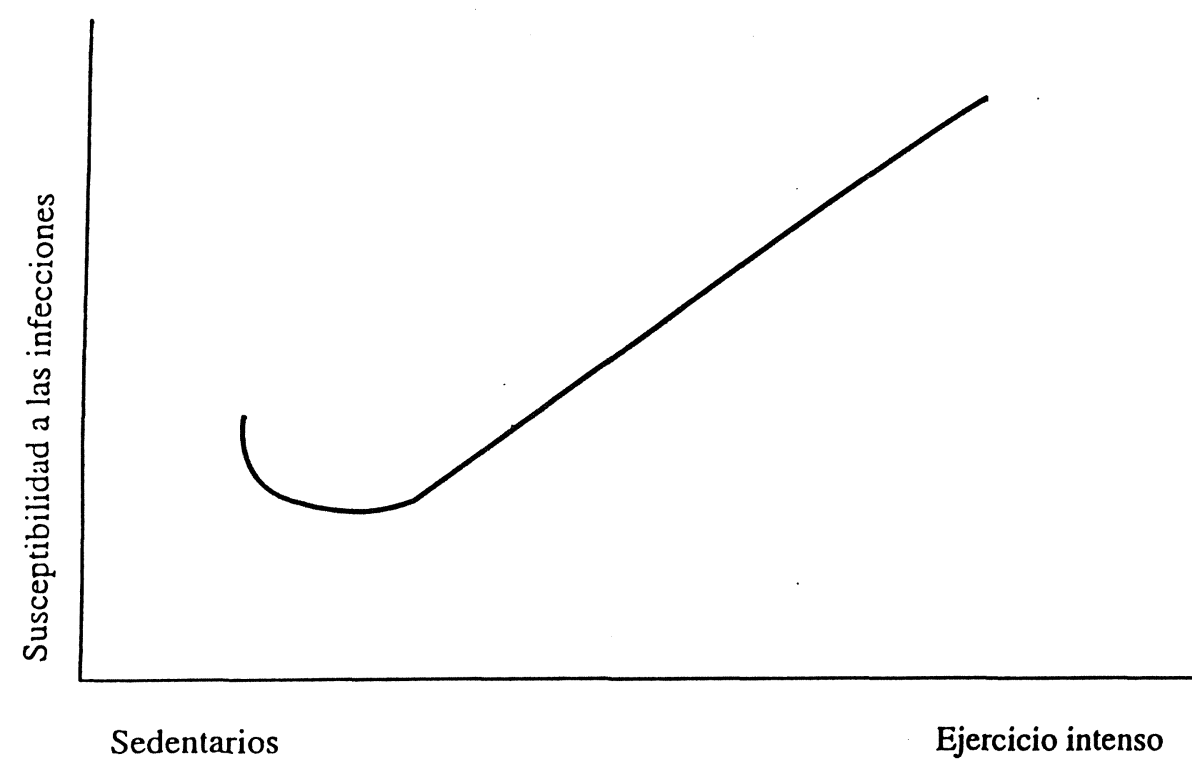


El entrenamiento intenso induce cambios negativos en la inmunidad mediados por los efectos en el músculo esquelético del cortisol, la adrenalina y los reactantes de fase aguda. El estrés psicológico es otro factor modulador. El ejercicio moderado, por el contrario, produce cambios que mejoran la protección inmunitaria ${ }^{4}$.

Existen datos epidemiológicos que confirman que el entrenamiento intenso aumenta la prevalencia y la persistencia de síntomas respiratorios lo que soporta la teoría actual de que el ejercicio intenso altera la respuesta inmunitaria ${ }^{16}$.

\section{Ejercicio y bienestar psicológico}

La realización de actividad física atenúa las respuestas fisiológicas originadas por el estrés, mejorando la sintomatología psíquica acompañante ${ }^{10}$. Se ha podido comprobar que el ejercicio físico tiene un efecto positivo en el humor y el bienestar mental, puede ayudar a manejar más eficazmente las situaciones de estrés y a recuperarse de los efectos negativos de los sucesos adversos de la vida diaria, reduce la ansiedad y la depresión y mejora la propia imagen. Estos beneficios se derivan, entre otras razones, de la reducción de las catecolaminas circulantes y la mejora del sueño, así como la liberación de endorfinas ${ }^{4,5,17}$. Se ha comprobado que los individuos con menor nivel de ejercicio inicial consiguen las mayores reducciones de ansiedad ${ }^{4}$. El ejercicio físico, sólo o asociado a la psicoterapia y a la farmacoterapia, se emplea en el tratamiento de diversos trastornos mentales ${ }^{10}$. De hecho, la actividad física se emplea en la actualidad como arma terapéutica adicional en la depresión ${ }^{5}$. Marcos et al en un estudio de 1980 advirtieron que el ejercicio físico redujo los síntomas acompañantes de la depresión y la ansiedad, mejoró la convivencia entre los enfermos, las relaciones entre éstos y sus cuidadores y el sueño, a la vez que contribuyó a la reducción del consumo de fármacos ${ }^{10}$.

\section{Recomendación de la práctica de ejercicio}

El médico de familia es la primera fuente de información médica en la mayoría de los casos ${ }^{12}$. Por tanto, los médicos están en una posición de privilegio para animar a sus pacientes a incrementar sus niveles de actividad física ${ }^{12}$. A pesar de ello la mayoría de los médicos no aconsejan a sus pacientes sobre el ejercicio físico. Sin embargo, los médicos juzgan 


\section{Influencia de la actividad física en la salud humana}

necesario aconsejar sobre la actividad física pero reconocen que, en líneas generales, no están preparados para ello. Quizás por este entrenamiento insuficiente, los médicos indican que sería de ayuda la existencia de protocolos de consejo sobre hábitos saludables.

Se debe sugerir incrementar la marcha o la actividad física general en la vida diaria ${ }^{7,13}$. Cada persona debería acumular 30 minutos o más de actividad física de moderada intensidad en la mayoría de los días de la semana ${ }^{1,13}$.

Para conseguir una máxima eficacia en la terapia de mejora de la salud, la prescripción de ejercicio debe cumplir una serie de requerimientos básicos: la actividad física debe abarcar la mayoría de los días de la semana (al menos cinco), ha de ser divertida, no debe ser dolorosa o extenuante y estará acorde con las preferencias individuales ${ }^{5}$. El ejercicio seleccionado debe ser llevado a cabo fácilmente y realizable próximo al domicilio o al lugar de trabajo. El equipamiento y las ropas deportivas no deberán ser caros. La actividad no deberá requerir la participación de otras personas (p.ej: deportes en equipo) pero puede permitir la realización en grupo, si se desea. Finalmente, la práctica deportiva elegida debe ser compatible con la realización continuada durante toda la vida ${ }^{5}$.

No se debe olvidar que hay que incentivar adecuadamente a los pacientes a adoptar programas de ejercicio físico. La gente necesita escuchar los beneficios del ejercicio ${ }^{13}$. Por ello, es aconsejable adoptar ciertas estrategias. Se debe preguntar a los pacientes por sus hábitos de ejercicio físico y relacionar las sugerencias de práctica de ejercicio físico con síntomas o quejas importantes (p.ej: «estoy gordo» o «me encuentro cansado»). Se ha de suministrar material de lectura acerca de los efectos beneficiosos del ejercicio. El cumplimiento de los programas de actividad física debe ser seguido en el tiempo por los médicos ${ }^{13}$.

Además del ejercicio, es conveniente aconsejar sobre otros hábitos saludables como una dieta adecuada. Se debe intentar consumir 2000 calorías a la semana que es el nivel óptimo para conseguir rebajar el riesgo de enfermedad cardiovascular. Si bien mayores gastos calóricos no ofrecen mayor protección cardiaca si que ayudan a perder peso y a sentirse mejor ${ }^{18}$.

El ejercicio físico adoptado debe ser aeróbico y moderadamente intenso. El objetivo es conseguir la frecuencia cardiaca basal más baja posible. La variedad en la práctica deportiva es importante en la prescripción.

En las personas mayores que no practicaron deporte previamente la adopción y el mantenimiento de un programa constante de marcha 
es la clave para conseguir una serie de beneficios en la salud, tales como, perdida de peso y disminución de la presión arterial. La perdida de peso puede reducir el riesgo de padecer diabetes y la necesidad de medicación. La marcha regular, puede también enlentencer el proceso de envejecimiento manteniendo los músculos, los huesos y las articulaciones fuertes y sanas. $\mathrm{Y}$ la marcha es un ejercicio simple. No es necesario utilizar pesas ni aparatos ${ }^{19}$.

\section{Bibliografía}

1 ACSM American College of Sports Medicine. Physical activity, physical fitness and hypertension, position stand. Med Sci Sports Exerc 1993;25(10):i-x.

2 ARTAL, R. Exercise: An alternative therapy for gestational diabetes. Phys Sportsmed 1996;24(3):54-66.

3 Bernstein, L.; Henderson, BE.; Hanisch, R. et al.: Physical exercise and reduced risk of breast cancer in young women. J Natl Cancer Inst 1994; 86(18):1403-8.

4 Bouchard, C.; Shephard, RJ.; Stephens, T.: Physical Activity and Fitness in Disease. En Bouchard C, Shephard RJ, Stephens T directores. Physical Activity, Fitness and Health. Champaign, Illinois: Human Kinetics, 1993; 61-83.

5 ELRICK, H.: Exercise is Medicine. Phys Sportsmed 1996;24(2):72-78.

6 ERIKSSON, JG.: Exercise and the treatment of type 2 diabetes mellitus. An update. Sports Med 1999;27(6):381-91.

7 Franklin, BA.; Blair, SN.; HASKell, WL.; ThompSON, P.D.; VAN CAMP, S.P.: Exercise and cardiac complications. Do the benefits outweigh the risks? Phys Sportsmed 1994;22(2):56-68.

8 HARGARTEN, K.M.: Menopause. How exercise mitigates symptoms. Phys Sportsmed 1994; (22)1:49-58.

9 LAKKA, T.A.; VENÄLÄINEN, J.M.; RAURAMAA, R. et al.: Relation of leissure-time physical activity and cardiorespiratory fitness to the risk of acute myocardial infarction in men. New Eng J Med 1994;330(22):1549-54.

10 MARCoS, J.F.: Las relaciones entre la salud, el ejercicio y la forma física. En: Marcos, J.F. director. Ejercicio. Forma Física y Salud. Madrid: Eurobook, 1994;17-58.

11 Mink, B.D.; DiNubILE, N.A.: Exercise and Chronic Obstructive Pulmonary Disease. Modest fitness gains pay big dividends. Phys Sportsmed 1997;25(11):43-52.

12 Patrick, K.; Sallis, J.F.; Long, B.; Calfas, K.J.; WoOten, W.; Heath, G.; PratT, M.: A new tool for encouraging activity. Project PACE. Phys Sporstmed 1994;22(11):45-55.

13 Potera, C.: Fighting the fitness slump. Phys Sportsmed 1994;22(1):49-58.

14 Potera, C.: Exercise and cancer risk. Phys Sportsmed 1995;23(1): 37-8.

15 Shangold, M.M.: An active menopause. Using exercise to combat symtoms. Phys Sportsmed 1996;24(7):30-36.

16 ShePhaRd, R.J.; PANG, N.S.; DiNuBILE, N.A.: Exercise, Inmunity and susceptibility to infection. Phys Sportsmed 1999; 27(6):47-71.

17 Sherman, C.: Stress: How to help patients cope. Phys Sportsmed 1994; $22(7): 66-75$ 


\section{Influencia de la actividad física en la salud humana}

18 STAMFORD, B.: Burning Calories-Naturally. Phys Sportsmed 1994;22(4):115.

19 STAMFORD, B.: Mall walking. Burning calories in the great indoors. Phys Sportsmed 1994;22(12):101.

20 TAunton, J.E.; MCCARgar, L.: Managing Activity in Patients who have diabetes. Practical ways to incorparte exercise in lifestyle. Phys Sportsmed 1995;23(3): 41-52.

21 WALBERG-Heriksson, H.: Exercise and Diabetes mellitus. Exerc Sport Sci Rev 1992;20:339-68. 